\title{
Selección de funciones de distribución de frecuencias diamétricas, para Pinus taeda, Eucalyptus globulus y Eucalyptus dunnii en Uruguay
}

\author{
Selecting diametric frequency distribution functions for Pinus taeda, \\ Eucalyptus globulus and Eucalyptus dunnii in Uruguay
}

\author{
Andrés Hirigoyen ${ }^{\text {a*, }}$ Cecilia Rachid ${ }^{\text {a }}$ \\ *Autor de correspondencia: a Instituto Nacional de Investigación Agropecuaria, Programa de Nacional Forestal, \\ Ruta 5 km 386 Tacuarembó, Uruguay, tel.: +59846323969, ahiriogoyen@tb.inia.org.uy
}

\begin{abstract}
SUMMARY
The aim of this study was to compare the accuracy of Weibull, reverse Weibull, $\mathrm{S}_{\mathrm{B}}$ Johnson, Gamma and Beta distributions, fitted with the method of moments and maximum likelihood, for describing diameter distributions in stands of Pinus taeda, Eucalyptus globulus, and Eucalyptus dunnii in Uruguay. Data from 429 permanent plots of P. taeda, 185 plots of E. globulus and 271 plots of E. dunnii were used. To compare the quality of fit for the probability distribution function (pdf), the Kolmogorov-Smirnov and Reynolds error indices were used. The function $\mathrm{S}_{\mathrm{B}}$ Johnson presented a better fit on the plots of $P$. taeda, while the inverted Weibull function was more accurate for Eucalyptus species. The method of moments was more accurate than maximum likelihood.
\end{abstract}

Key words: pdf, distribution, Weibull, $\mathrm{S}_{\mathrm{B}}$ de Johnson.

\section{RESUMEN}

El objetivo de este estudio fue comparar la precisión de las distribuciones de Weibull, Weibull reversa, $\mathrm{S}_{\mathrm{B}}$ de Johnson, Gamma y Beta, ajustadas por método de los momentos y máxima verosimilitud, para describir distribuciones diamétricas de Pinus taeda, Eucalyptus globulus y Eucalyptus dunnii en Uruguay. Se utilizaron datos de un total de 429 parcelas permanentes de P. taeda, 185 parcelas de E. globulus y 271 parcelas de E. dunnii. Para compararla calidad del ajuste de las funciones de densidad de probabilidad (fdp) se utilizaron la prueba de Kolmogorov-Smirnov y el índice de error de Reynolds. La función $\mathrm{S}_{\mathrm{B}}$ de Johnson presentó un mejor ajuste en las parcelas de $P$. taeda, mientras la función Weibull reversa fue más precisa para las especies de Eucalyptus. El método de los momentos resultó con mayor precisión que el método de máxima verosimilitud.

Palabras clave: fdp, distribución, Weibull, $\mathrm{S}_{\mathrm{B}}$ de Johnson.

\section{INTRODUCCIÓN}

Conocer el número de árboles correspondiente a cada clase diamétrica de un rodal es de gran utilidad para el planeamiento y manejo de plantaciones, ya que permite estimar la variabilidad entre individuos, los posibles productos a obtener y, por lo tanto, el valor de la plantación. Las funciones de densidad de probabilidad (fdp) son utilizadas en el ámbito forestal para estimar la probabilidad de ocurrencia de un número de individuos y la probable distribución que presentará según sus diámetros, describiendo en términos matemáticos la estructura del rodal (Vallejos et al. 2004).

Dentro de las fdp más utilizadas se destacan las funciones de $\mathrm{S}_{\mathrm{B}}$ de Johnson, Weibull, Weibull reversa, Beta y Gamma, siendo las distribuciones de Weibull y $S_{B}$ de Johnson las más empleadas debido a una mejor calidad de ajuste (Vanclay 1994). Sanquetta et al. (2014) trabajaron con las funciones Gamma, Log Normal, Normal y Weibull (dos y tres parámetros), para describir las distribuciones diamétricas de Acacia mearnsii De Wild.. La función de Weibull es ampliamente utilizada en el ámbito forestal, su versión reversa (Kuru et al. 1992, Xu et al. 1992), en la cual el origen de la curva de distribución diamétrica se cambia del diámetro a la altura del pecho (DAP) mínimo al máximo, presenta como ventajas: a) mayor facilidad para medir, estimar y proyectar DAP máximo frente al DAP mínimo, b) los árboles pertenecientes a la clase de DAP máximo representan el mayor valor del rodal (las estimaciones en esa zona de la distribución tiene mayor relevancia que las clases diamétrica mínimas), c) luego de un raleo selectivo por lo alto el DAP mínimo se ve alterado mientras que se asume que el DAP máximo no sufre cambios (Methol 2003).

Vanclay (1994) plantea que la estimación de los parámetros de las fdp se puede realizar por varios métodos, diferenciándolos entre métodos de recuperación y métodos de predicción de parámetros. En la recuperación de parámetros se busca relacionar variables del rodal futuro con 
momentos o percentiles de la distribución esperada. Para el cálculo de parámetros, se emplean métodos como: máxima verosimilitud (Bailey y Dell 1973), percentiles y momentos (Scolforo 2002, Maestri 2003, Vallejos et al. 2011). En los métodos de predicción se modelan los estimadores de los parámetros de la fdp mediante modelos lineales, usando como variables predictoras el índice de sitio, altura total o edad, entre otras. El mismo tiene la desventaja de que las predicciones de variables como área basal y volumen realizadas a través de la predicción de parámetros no son compatibles con valores obtenidos mediante la predicción directa de estas variables (Burkhart y Tomé 2012).

Existen varias pruebas disponibles para evaluar la bondad de ajuste de la distribución estimada con respecto a la observada, siendo la prueba de Kolmogorov-Smirnov y la prueba $\chi^{2}$ de Pearson los más utilizados (Scolforo2002). Para esta última es necesario reagrupar los datos en al menos cinco clases de DAP, cada una con una frecuencia absoluta mayor o igual a tres. Por otro lado, la prueba de adherencia de Kolmogorov-Smirnov presenta mayor sensibilidad a las variaciones de la distribución teórica (Gibbons y Chakraborti 1992). Otro índice utilizado para evaluar la adherencia de las fdp es el índice de error (Reynolds et al.1988), el mismo indica la diferencia entre la frecuencia estimada por la fdp y la real.

En Uruguay, existen escasos antecedentes de estudios sobre funciones de densidad de probabilidad; se ha estudiado la función de Weibull reversa para modelar la distribución diamétrica en plantaciones de Eucalyptus grandis Hill ex Maiden (Methol 2001 y 2003) y también se ha ajustado esta función para Eucalyptus globulus Labill (Methol 2006). Sin embargo, no existen funciones ensayadas para otras especies de uso común como Pinus taeda L. así como tampoco existen estudios conocidos sobre el uso de otras funciones. Con base en estos antecedentes nacionales se plantea la hipótesis que: i) existe una pdf que se ajusta a cada especie en estudio y ii) explica de forma certera la distribución de los árboles en pie según su DAP.

Los objetivos de este trabajo son: a) comparar la calidad de ajuste de las distribuciones de Weibull, Weibull reversa, $S_{B}$ de Johnson, Beta y Gamma ajustadas por uno o ambos de los siguientes métodos: (a.i) método de los momentos y (a.ii) de máxima verosimilitud; b) seleccionar la fdp que mejor se ajuste a la distribución diamétrica de parcelas de Pinus taeda, Eucalyptus globulus y Eucalyptus dunnii en Uruguay.

\section{MÉTODOS}

Para el ajuste de las fdp de interés se utilizaron 885 parcelas permanentes de plantaciones situadas en los departamentos de Durazno, Lavalleja, Rocha, Tacuarembó y Rivera en Uruguay. Los datos descriptivos de las parcelas inventariadas se incluyen en el cuadro 1.

Para cada parcela se calculó el diámetro máximo, diámetro mínimo, diámetro promedio y desvío estándar, variables necesarias para estimar los parámetros de las fdp
Cuadro 1. Descripción de las parcelas utilizadas para el estudio. Description of plots used in the study.

\begin{tabular}{lrcc}
\hline \multicolumn{1}{c}{ Característica } & $\begin{array}{c}\text { Pinus } \\
\text { taeda }\end{array}$ & $\begin{array}{c}\text { Eucalyptus } \\
\text { glubulus }\end{array}$ & $\begin{array}{c}\text { Eucalyptus } \\
\text { dunnii }\end{array}$ \\
\hline Parcelas totales & 429 & 185 & 271 \\
Edad mínima (años) & 2 & 3 & 3 \\
Edad máxima (años) & 26 & 13 & 12 \\
DAP máximo (cm) & 45 & 35 & 38 \\
DAP mínimo (cm) & 2,5 & 2 & 3 \\
\hline
\end{tabular}

estudiadas a través de la recuperación de parámetros. En las fdp de Weibull, $\mathrm{S}_{\mathrm{B}}$ Johnson y Gamma los parámetros fueron estimados por el método de los momentos y máxima verosimilitud, mientras que para la Weibull reversa y Gamma se utilizó el método de los momentos. Las metodologías aplicadas fueron las utilizadas por Nelson (1964), Johnson y Kitchenl (1971), Methol (2001), Scolforo (2002) y Vallejos et al. (2004).

Las funciones comparadas, así como sus métodos de ajuste se describen a continuación.

Función de Weibull. La función Weibull se define como:

$$
f(D A P)=\frac{c}{b}\left(\frac{D A P-a}{b}\right)^{c-1} e^{\left(-\left(\frac{D A P-a}{b}\right)^{c}\right)}
$$

Donde: $f(\mathrm{DAP})$ es la probabilidad de ocurrencia o frecuencia del DAP de interés (adquiere valores comprendidos entre 0 y 1); $a$ es el parámetro de localización; $b$ es el parámetro de escala; y c es el parámetro de forma de la distribución.

El parámetro $a$ se calcula mediante procesos iterativos como cierto porcentaje del diámetro mínimo comprendido entre el $5 \%$ y el $100 \%$ (Maestri 2003, Eisfeld 2004). Vallejos et al. (2004) sugieren igualar este parámetro a cero y utilizar una versión de Weibull trunca.

La estimación de parámetros para esta función fue realizada por método de máxima verosimilitud (Vallejos et al.2004) de la siguiente forma:

$$
\begin{gathered}
c=\left[\frac{\mathrm{n}}{\left.\frac{\sum_{\mathrm{i}=1}^{\mathrm{n}}(\mathrm{DAP}-\mathrm{a})^{\mathrm{c}} \ln (\mathrm{DAP}-\mathrm{a})}{\sum_{\mathrm{i}=1}^{\mathrm{n}(\mathrm{DAP}-\mathrm{a})^{\mathrm{c}}}-\sum_{\mathrm{i}=1}^{\mathrm{n}} \ln (\mathrm{DAP}-\mathrm{a})}\right]}\right. \\
b=\frac{\left(\sum_{i=1}^{n}(D A P-a)^{c}\right)^{\frac{1}{c}}}{n^{\frac{1}{c}}}
\end{gathered}
$$

Donde: $n$ es el número de observaciones y ln es logaritmo natural.

Para la misma fdp también se ensayó la estimación de parámetros por método de los momentos (Vallejos et 
al. 2004) como se describe a continuación:

$$
\begin{aligned}
& \frac{\sigma_{d a p-a}}{\overline{D a p}-a}=\frac{\sqrt{\Gamma\left(1+\frac{2}{c}\right)-\Gamma^{2}\left(1+\frac{1}{c}\right)}}{\Gamma\left(1+\frac{1}{c}\right)} \\
& b=\frac{\overline{D a p}-a}{\Gamma\left(1+\frac{1}{c}\right)}
\end{aligned}
$$

Donde: $\sigma_{\text {dap }}$ desvío estándar de los DAP; $\overline{D a p}$ diámetro medio aritmético; $\Gamma$ función Gamma.

Función de Weibull reversa. La función se formula como:

$$
f(D A P)=(c / b)\left(\frac{a-D A P}{b}\right)^{c-1} \exp \left[-\left(\frac{a-D A P}{b}\right)^{c}\right][6]
$$

Donde: $a$ es el parámetro que indica el punto de partida de la distribución (DAP máximo); $b$ es el parámetro de escala; y c es el parámetro de forma de la distribución.

La estimación de parámetros mediante el método de los momentos (Methol 2001) es la siguiente: $a=$ DAP máximo.

$$
\begin{gathered}
b=\frac{a-\overline{D A P}}{\Gamma\left(1+\frac{1}{c}\right)} \\
c=\frac{1}{z\left[1+(1-z)^{2}(k z 0+k z 1+k z 2+k z 3+k z 4+k z 5)\right]} \\
z=\frac{\sigma_{\text {dap }}}{a-\overline{D A P}}
\end{gathered}
$$

Donde: $\sigma_{\text {dap }}$ es el desvío de los DAP; $k$ el coeficiente propuesto por García (1981).

Función de $S_{B}$ de Johnson. La función $\mathrm{S}_{\mathrm{B}}$ de Johnson se expresa como:

$$
\begin{aligned}
f(D A P)= & \frac{\delta}{\sqrt{2 \pi}}\left(\frac{\lambda}{(D A P-\varepsilon)(\lambda+\varepsilon-D A P)}\right) \\
& \exp \left\{-\frac{1}{2}\left[\gamma+\delta \ln \left(\frac{\delta-\varepsilon}{\lambda+\varepsilon-D A P}\right)\right]^{2}\right\}
\end{aligned}
$$

Donde: $\varepsilon$ es el parámetro de localización; $\lambda$ es el parámetro de escala; $\delta$ y $\gamma$, representan la kurtosis y el parámetro de asimetría de la distribución, respectivamente.

La estimación de parámetros por método de los momentos (Johnson y Kitchenl1971) es la siguiente:

$$
\begin{gathered}
\delta=\frac{\mu(1-\mu)}{S d(x)}+\frac{S d(x)}{4}\left[\frac{1}{\mu(1-\mu)}-8\right] \\
\gamma=\delta \ln \left(\frac{1-\mu}{\mu}\right)+\left(\frac{0,5-\mu}{\delta}\right)
\end{gathered}
$$

$$
\begin{gathered}
\mu=\frac{\overline{D A P}-\varepsilon}{\lambda} \\
S d(x)=\frac{\sigma_{d a p}}{\lambda}
\end{gathered}
$$

Donde: $S d(x)$ es el desvío estándar modificado; $\sigma_{\text {dap }}$ el desvío estándar de la parcela, $\overline{D A P}$ el DAP medio aritmético, $\lambda$ el DAP máximo y $\varepsilon$ el $\%$ de DAP mínimo.

La estimación de parámetros por método de máxima verosimilitud (Scolforo 2002) para esta función es:

$$
\begin{gathered}
f_{i}=\ln \left(\frac{d_{i}-\varepsilon}{\varepsilon+\lambda-d_{i}}\right) \\
\sigma_{f}=\sqrt{\frac{\sum_{i=1}^{n} f_{i}^{2}-\frac{1}{n}\left(\sum_{i=1}^{n} f_{i}\right)^{2}}{n-1}} \\
\delta=\frac{1}{\sigma f} \\
\gamma=-\frac{\bar{f}}{\sigma f}
\end{gathered}
$$

Donde: $=\sigma_{f}$ desvío estándar de la variable $f_{\mathrm{i}} ; n=$ número de observaciones; $d_{\mathrm{i}}=$ diámetro.

Función Beta. La función Beta se define como:

$$
f(\text { dap })=\frac{\Gamma(\alpha+\beta)}{\Gamma(\alpha) \Gamma(\beta)} \frac{(\mathrm{DAP}-\mathrm{a})^{\alpha-1}(\mathrm{~b}-\mathrm{DAP})^{\beta-1}}{(\mathrm{~b}-\mathrm{a})^{\alpha+\beta-1}}
$$

Siendo y $a \leq D A P \leq b$ y $a>0$.

Donde: $a$ es el límite inferior (DAP mínimo), $b$ el límite superior de la distribución (DAP máximo), $\alpha$ y $\beta$ parámetros de la distribución (obtenidos por método de estimación) (Scolforo 2002).

Los parámetros para esta función se estiman de la siguiente manera por método de los momentos (Scolforo 2002):

$$
\begin{gathered}
\alpha=\frac{(\overline{D A P}-a)\left((b-\overline{D A P})(\overline{D A P}-a)-\sigma^{2}\right)}{\sigma^{2}(b-a)} \\
\beta=\frac{(b-\overline{D A P})\left((b-\overline{D A P})(\overline{D A P}-a)-\sigma^{2}\right)}{\sigma^{2}(b-a)}
\end{gathered}
$$

Donde: $\sigma^{2}=$ varianza de los DAP; $a$ y $b=D A P$ mínimo y máximo, respectivamente.

Función Gamma. La función Gamma se formula como:

$$
f(D A P)=\frac{D A P^{\alpha-1} e^{-\frac{D A P}{\beta}}}{\beta^{\alpha} \Gamma(\alpha)}
$$


Donde: $\alpha$ y $\beta$ parámetros de la distribución (obtenidos por método de estimación), $\Gamma$ función Gamma.

Por el método de los momentos (Scolforo 2002) los parámetros se estiman de acuerdo a las siguientes expresiones:

$$
\begin{gathered}
\alpha=\frac{\overline{D A P}^{2}}{\sigma^{2}} \\
\beta=\frac{\sigma^{2}}{\overline{D A P}}
\end{gathered}
$$

Donde: $\sigma^{2}=$ varianza de los DAP; $\overline{D A P}=D A P$ medio de la muestra.

Mientras que por método de máxima verosimilitud (Nelson 1964) los parámetros se estiman de la siguiente forma:

$$
\begin{gathered}
\alpha=\frac{1+\sqrt{1+\frac{4}{3}\left(\ln (\overline{D A P})-\frac{1}{n} \sum_{i=1}^{n} \ln \left(d_{i}\right)\right)}}{4\left(\ln (\overline{D A P})-\frac{1}{n} \sum_{i=1}^{n} \ln \left(d_{i}\right)\right)} \\
\beta=\frac{\overline{D A P}}{\alpha}
\end{gathered}
$$

Donde: $\overline{D A P}=$ DAP medio de la muestra, $\ln \left(d_{\mathrm{i}}\right)=$ logaritmo natural del i-ésimo diámetro de la muestra $(\mathrm{i}=1,2, \ldots, \mathrm{n})$, $\mathrm{n}$ = número de observaciones.

Como medida de bondad del ajuste de las fdp se utilizaron: la prueba de Kolmogorov-Smirnov (mediante el estadístico $d n$ ), y el índice de error (IE) propuesto por Reynolds et al. (1988). Ambas pruebas comparan la distribución teórica obtenida de las diferentes fdp con la distribución real. La prueba de Kolmogorov-Smirnov define su estadístico, $d n$, con base en la diferencia máxima (punto de mayor divergencia), entre la distribución acumulada teórica y la observada (Newnham 1992, Gadow1983). La hipótesis nula de que la distribución esperada es similar a la observada se rechaza para valores de $d n$ altos comparados con un valor critico $(d)$ (para $\mathrm{P}(d n \geq d),=\alpha, \alpha=0,05)$ :

$$
d n=\operatorname{Max}[S(x)-F(x)]
$$

Donde: $F(x)$ valor de la función de la distribución de frecuencia acumulada teórica; $S(x)$ valor de la función de distribución de frecuencia acumulada observada, $d$ valor de tabla para $\alpha=0,05$.

Se utilizó el porcentaje de parcelas rechazadas por esta prueba, para cada una de las fdp (según el método de estimación de parámetros previamente seleccionado) como criterio de selección de las fdp. El índice de error (Reynolds et al.1988), indican la cantidad de árboles erróneamente estimados por clases diamétricas. A menor valor del índice de error mejor será el método de estimación. Su fórmula de cálculo es:

$$
I E=\sum_{j=1}^{k}\left|n_{j}-\widehat{n_{j}}\right|
$$

Donde: $I E$ es el índice de error, $n_{j}$ es el número de árboles reales en la clase diamétrica $j, \hat{h}_{j}$,corresponde al número de árboles estimado por la fdp para la clase $j \mathrm{y}$ k es el total de clases diamétricas.

Todos los análisis y ajustes estadísticos se realizaron con el software SAS versión 8.0 (SAS 2007).

\section{RESULTADOS}

Para la pdf Weibul el valor de $a$ que incluyó más parcelas fue de $50 \%$ del DAP mínimo para las tres especies estudiadas (cuadro 2). También puede observarse que para esta función el método de los momentos incluyó más parcelas para las especies de Eucalyptus pero no para P. taeda.

En el caso de la función $S_{B}$, para obtener el valor del parámetro a través del método de los momentos y de máxima verosimilitud, se vinculó dicho parámetro al diámetro mínimo registrado en cada parcela. Se evaluaron porcentajes comprendidos entre el 5 y el $95 \%$, en intervalos de $5 \%$.

En ambos métodos los valores de $\varepsilon$ correspondientes a 25 y $30 \%$ del DAP mínimo presentaron los mayores porcentajes de parcelas incluidas para las tres especies, siendo el $30 \%$ el valor de mayor inclusión de parcelas para todas las especies y para ambos métodos (cuadro 3). A su vez el método de los momentos para los dos porcentajes seleccionados, incluyó más parcelas que el método de máxima verosimilitud.

Comparación de las distribuciones. Se observó que para $P$. taeda, la función $\mathrm{S}_{\mathrm{B}}$ fue la mejor posicionada, según $d n$, tanto por el método de momentos como por máxima verosimilitud (cuadro 4). Para E. dunnii, la función Weibull fue la mejor puntuada por ambos métodos, al incluir la función Weibull reversa ajustada por el método de los momentos, esta fue la que mostró menor número de parcelas rechazadas, incluso a nivel del ranking general $\left(\mathrm{R}_{3}\right)$ (cuadro 4). Finalmente, para E. globulus el ranking según ambos métodos apunto a la función Weibull como la mejor función ajustada por el método de los momentos, mientras que por máxima verosimilitud fue la función $\mathrm{S}_{\mathrm{B}}$ la mejor posicionada (cuadro 4). Por otro lado, incluyendo las funciones ajustadas solamente por método de los momentos $\left(R_{2}\right)$ la función Weibull reversa fue la de mejor ranking, posicionándose como la mejor también en el ranking general $\left(\mathrm{R}_{3}\right)$.

En general, las fdp ajustadas por método de máxima verosimilitud presentaron menor índice de error (cuadro 5), con poca diferencia con respecto al método de los momentos.

Para $P$. taeda, el ranking por índice de error se mantuvo con respecto al ranking general del cuadro $4\left(\mathrm{R}_{3}\right)$, con diferencia que en este último las funciones Weibull y Weibull reversa se ubicaron en las posiciones 2 y 3 , mientras que en el ranking por índice de error se invierten. En E. globulus 
Cuadro 2. Número de parcelas con frecuencia estimada similar a la observada, cuando $a$ adquiere valores del $50 \%$ del DAP mínimo para la función Weibull.

Number of plots with similar estimated and observed frequency values, where $a$ represents $50 \%$ of the minimum DBH for the Weibull function.

\begin{tabular}{lccc}
\hline \multicolumn{1}{c}{ Método } & $\begin{array}{c}\text { Pinus } \\
\text { taeda }\end{array}$ & $\begin{array}{c}\text { Eucalyptus } \\
\text { glubulus }\end{array}$ & $\begin{array}{c}\text { Eucalyptus } \\
\text { dunnii }\end{array}$ \\
\hline Momentos & 406 & 258 & 173 \\
Máxima verosimilitud & 412 & 256 & 150 \\
\hline
\end{tabular}

Prueba Kolmogorov-Smirnov $\alpha=0,05$.

Cuadro 3. Número de parcelas con frecuencia estimada similar a la observada según (porcentaje del DAP mínimo) para la función $\mathrm{S}_{\mathrm{B}}$.

Number of plots with similar estimated and observed values by $\varepsilon$ (percentage of the minimum $\mathrm{DBH}$ ) for the function $\mathrm{S}_{\mathrm{B}}$

\begin{tabular}{lcccccc}
\hline \multirow{2}{*}{ Método } & \multicolumn{2}{c}{$\begin{array}{c}\text { Pinus } \\
\text { taeda }\end{array}$} & $\begin{array}{c}\text { Eucalyptus } \\
\text { glubulus }\end{array}$ & $\begin{array}{c}\text { Eucalyptus } \\
\text { dunnii }\end{array}$ \\
\cline { 2 - 7 } & $\varepsilon=25 \% \varepsilon=30 \%$ & $\varepsilon=25 \%$ & $\varepsilon=30 \%$ & $\varepsilon=25 \% \varepsilon=30 \%$ \\
\hline Momentos & 419 & 425 & 211 & 250 & 125 & 172 \\
$\begin{array}{l}\text { Máxima } \\
\text { verosimilitud }\end{array}$ & 309 & 423 & 205 & 237 & 101 & 159 \\
\hline
\end{tabular}

Prueba Kolmogorov-Smirnov $\alpha=0,05$

ocurrió lo mismo para las funciones Weibull y $\mathrm{S}_{\mathrm{B} \text {, }}$ en $\mathrm{R}_{3}$ estaban en segundo y tercer lugar, mientras que por índice de error se invierten. En cambio para E. dunnii coincidieron solamente el primero y el último lugar, mientras que las posiciones intermedias cambiaron. La figura 1 presenta como ejemplo el ajuste de las fdp según el método previamente seleccionado para cada especie, para tres parcelas.

\section{DISCUSIÓN}

Coincidiendo con los resultados obtenidos, Hawkins et al. (1988) y Liu et al.(2004) reportan como el valor más frecuentemente utilizado para en la fdp Weibull, el $50 \%$ del DAP mínimo. Gorgoso et al. (2012) proponen el mismo valor para dicha fdp (ajustada para P. pinaster, P. radiata y $P$. sylvestris). Según Scolforo y Thierschi (1998), trabajando con E. camaldulensis, y Abreu et al. (2002) con E. grandis, en la fdp $\mathrm{S}_{\mathrm{B}}$, el valor del parámetro con el cual obtuvieron resultados satisfactorios fue del $5 \%$.

Los resultados del ajuste de las distribuciones a través de la evaluación del estadístico $d n$, demuestran que el método de los momentos presenta un mejor ajuste con un porcentaje mayor del total de las parcelas evaluadas para ambos valores en ambas fdp (Weibull y $\mathrm{S}_{\mathrm{B}}$ ) para las especies de eucaliptus. La fdp Weibull para P. taeda presenta mayor porcentaje de adherencia en la estimación por máxima verosimilitud.
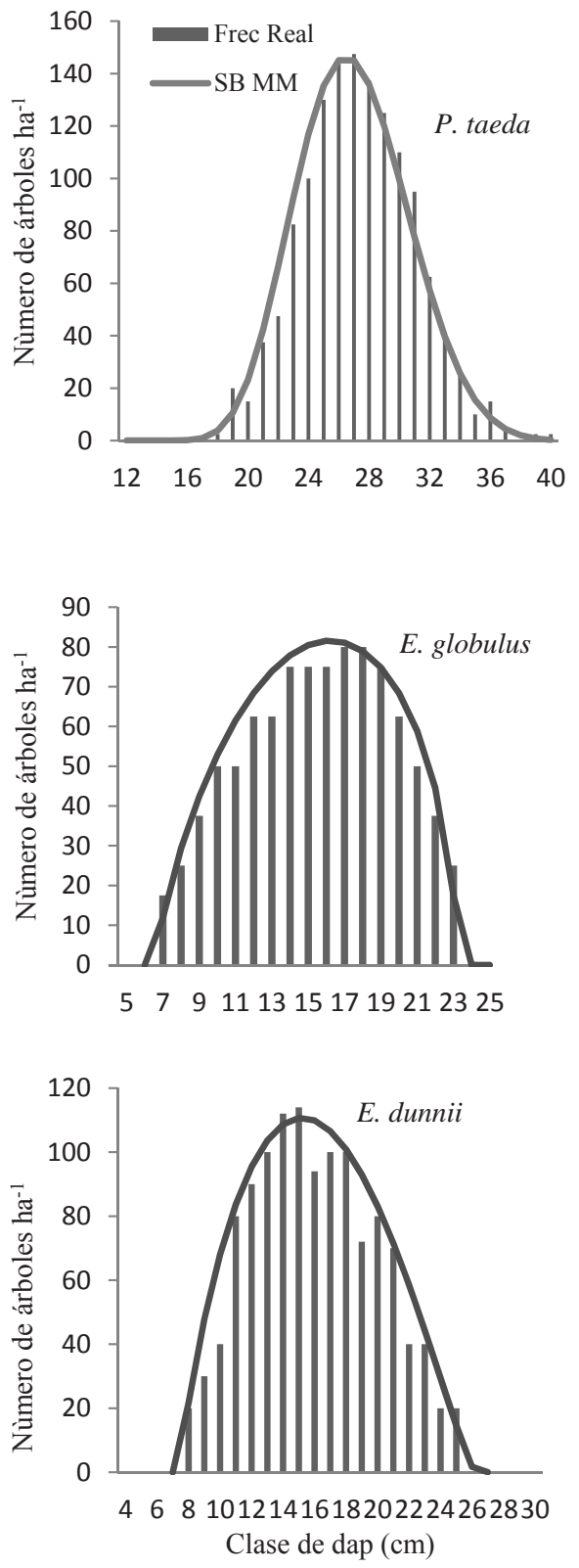

Figura 1. Ajuste para tres fdp según el método previamente seleccionado. Frec Real: frecuencia real, SB MM: distribución $S_{B}$ de Johnson, W. reversa: distribución de Weibull reversa.

Three pdf adjusted according to the previously selected method.

Según el ranking por método, $\mathrm{R}_{1}$ (distribuciones ajustadas por ambos métodos), hay acuerdo de la mejor ecuación para P. taeda y E. dunnii pero no para E. globulus. Para esta última, diferentes métodos apuntaron a diferentes distribuciones, sin embargo, sí hay consistencia respecto a la peor distribución. Gamma y Beta obtuvieron, en general, los últimos lugares en $\mathrm{R}_{3}$, para las tres especies, coincidiendo con los resultados obtenidos por Stepka et al. (2011). Sólo para E. dunnii el ranking de distribuciones fue igual para ambos métodos de estimación. En general, el método de los momentos muestra el mínimo valor de parcelas recha- 
Cuadro 4. Número, porcentaje y rankings de parcelas rechazadas por especie según función y método de estimación. Number, percentage and rankings of rejected plots by species, function and estimation method.

\begin{tabular}{|c|c|c|c|c|c|c|c|c|c|c|c|c|c|c|c|}
\hline \multirow{2}{*}{ Distribución y método } & \multicolumn{5}{|c|}{ Pinus taeda } & \multicolumn{5}{|c|}{ Eucalyptus glubulus } & \multicolumn{5}{|c|}{ Eucalyptus dunnii } \\
\hline & Número & $\%$ & $\mathrm{R}_{1}$ & $\mathrm{R}_{2}$ & $\mathrm{R}_{3}$ & Número & $\%$ & $\mathrm{R}_{1}$ & $\mathrm{R}_{2}$ & $\mathrm{R}_{3}$ & Número & $\%$ & $\mathrm{R}_{1}$ & $\mathrm{R}_{2}$ & $\mathrm{R}_{3}$ \\
\hline Weibull momentos & 23 & 5,3 & 3 & 2 & - & 13 & 4,7 & 1 & 3 & 3 & 12 & 6,5 & 1 & 2 & 2 \\
\hline Weibull máxima verosimilitud & 17 & 3,9 & 2 & - & 2 & 15 & 5,6 & 1 & - & - & 35 & 18,9 & 2 & - & - \\
\hline $\mathrm{S}_{\mathrm{B}}$ momentos & 4 & 0.9 & 1 & 1 & 1 & 21 & 7.7 & 3 & 5 & 5 & 13 & 7,1 & 2 & 3 & 3 \\
\hline $\mathrm{S}_{\mathrm{B}}$ máxima verosimilitud & 6 & 1,4 & 1 & - & - & 34 & 12,5 & 3 & - & - & 26 & 14,1 & 1 & - & - \\
\hline Gamma momentos & 49 & 11,4 & 2 & 4 & 4 & 18 & 6,6 & 2 & 4 & - & 78 & 42,1 & 3 & 5 & - \\
\hline Gama máxima verosimilitud & 52 & 12,1 & 3 & - & - & 16 & 5,9 & 2 & - & 4 & 55 & 29,7 & 3 & - & 4 \\
\hline Beta momentos & 53 & 12,4 & - & 5 & 5 & 10 & 3,7 & - & 2 & 2 & 55 & 29,7 & - & 4 & 4 \\
\hline Weibull reversa momentos & 34 & 7,9 & - & 3 & 3 & 2 & 0,7 & - & 1 & 1 & 4 & 2,1 & - & 1 & 1 \\
\hline
\end{tabular}

$\mathrm{R}_{1}$ : Ranking para cada método (solo para ecuaciones ajustadas por dos métodos).

$\mathrm{R}_{2}$ : Ranking general por método de los momentos.

$\mathrm{R}_{3}$ : Ranking general (utilizando el método más ajustado para cada distribución).

Cuadro 5. Promedio, desvío estándar y valor máximo del índice de error de las fdp para cada especie, según método de estimación de parámetros.

Average, standard deviation and maximum value of error indices of pdf for each species, by the parameter estimation method.

\begin{tabular}{|c|c|c|c|c|c|}
\hline \multirow{2}{*}{ Especie } & \multirow{2}{*}{ Función } & \multirow{2}{*}{ Método } & \multicolumn{3}{|c|}{ Estadístico } \\
\hline & & & Promedio & Desvío & Máximo \\
\hline \multirow{8}{*}{ Pinus taeda } & \multirow{2}{*}{ Weibull } & $\mathrm{mv}$ & 30,8 & 45,3 & 752 \\
\hline & & $\mathrm{m}$ & 31,7 & 65,2 & 829 \\
\hline & \multirow{2}{*}{$\mathrm{S}_{\mathrm{B}}$} & $\mathrm{mv}$ & 4,14 & 6,75 & 139 \\
\hline & & $\mathrm{m}$ & 4,32 & 7,21 & 145 \\
\hline & \multirow{2}{*}{ Gamma } & $\mathrm{mv}$ & 31,5 & 66,2 & 946 \\
\hline & & $\mathrm{m}$ & 32,2 & 69,0 & 935 \\
\hline & Beta & $\mathrm{m}$ & 43,7 & 78,2 & 924 \\
\hline & Weibull reversa & $\mathrm{m}$ & 5,11 & 13,2 & 313 \\
\hline \multirow{8}{*}{ Eucalyptus dunnii } & \multirow{2}{*}{ Weibull } & $\mathrm{mv}$ & 2,00 & 1,59 & 13,1 \\
\hline & & $\mathrm{m}$ & 2,61 & 2,71 & 17,5 \\
\hline & \multirow{2}{*}{$\mathrm{S}_{\mathrm{B}}$} & $\mathrm{mv}$ & 3,59 & 3,72 & 28,1 \\
\hline & & $\mathrm{m}$ & 3,46 & 3,62 & 25,6 \\
\hline & \multirow{2}{*}{ Gamma } & $\mathrm{mv}$ & 2,60 & 2,70 & 17,5 \\
\hline & & $\mathrm{m}$ & 2,68 & 2,82 & 18,6 \\
\hline & Beta & $\mathrm{m}$ & 3,35 & 2,94 & 19,8 \\
\hline & Weibull reversa & $\mathrm{m}$ & 1,35 & 1,28 & 12,5 \\
\hline \multirow{8}{*}{ Eucalyptus globulus } & \multirow{2}{*}{ Weibull } & $\mathrm{mv}$ & 10,2 & 9,99 & 56,3 \\
\hline & & $\mathrm{m}$ & 12,3 & 10,1 & 63,4 \\
\hline & \multirow{2}{*}{$\mathrm{S}_{\mathrm{B}}$} & $\mathrm{mv}$ & 1,16 & 1,09 & 13,0 \\
\hline & & $\mathrm{m}$ & 1,15 & 1,09 & 132 \\
\hline & \multirow{2}{*}{ Gamma } & $\mathrm{mv}$ & 16,1 & 11,2 & 62,8 \\
\hline & & $\mathrm{m}$ & 16,6 & 12,1 & 66,2 \\
\hline & Beta & $\mathrm{m}$ & 27,3 & 13,2 & 57,6 \\
\hline & Weibull reversa & $\mathrm{m}$ & 0,19 & 5,9 & 25,5 \\
\hline
\end{tabular}

Mv: método de máxima verosimilitud, m: método de los momentos. 
zadas. Sin embargo, hubo excepciones: la distribución de Weibull ajustada para $P$. taeda y de Gamma ajustada para E. globulus y E. dunnii. Shiver (1988) observó iguales o menores diferencias entre las distribución esperada y observada a través del método de los momentos modificado en comparación con las estimaciones por máxima verosimilitud. Varios autores sugieren al método de los momentos como el mejor método de estimación debido a su simplicidad y precisión (Nanang 1998, Al-Fawzan 2000, Methol 2001).

La variación en importancia relativa de las diferentes distribuciones según el método de ajuste empleado también es mencionado por Zhang et al. (2003), quienes atribuyeron dicha variación a la exactitud con que diferentes métodos estiman los parámetros de cada distribución. Shiver (1988) observó mayor exactitud en la estimación de parámetros, utilizando máxima verosimilitud que mediante el método de los momentos.

En los rankings para todas las especies ajustadas por método de los momentos $\left(\mathrm{R}_{2}\right)$, las distribuciones que incluyeron más parcelas fueron $\mathrm{S}_{\mathrm{B}}$ para $P$. taeda y Weibull reversa para ambas especies de Eucalyptus. Glade (1986), Abreu et al. (2002) y Retslaff et al. (2012), trabajando con E. grandis, coinciden con la eficacia del método de los momentos para estimar los parámetros de $\mathrm{S}_{\mathrm{B}}$ y Weibull.

El ranking general (considerando el método con mayor inclusión de parcelas por distribución) $\mathrm{R}_{3}$, coincidió con los resultados del ranking $\mathrm{R}_{2}$.La mejor ecuación para $P$. taeda fue $\mathrm{S}_{\mathrm{B}}$ mientras que para $E$. dunnii y E. globulus fue Weibull reversa (esta distribución tampoco obtuvo una mala posición en este ranking para $P$. taeda). Las distribuciones con peor ajuste son Beta para $P$. taeda y E. globulus, y $\mathrm{S}_{\mathrm{B}}$ para E. dunnii. En general, la distribución Gamma es consistentemente peor posicionada entre las tres especies, mientras que la distribución Weibull se mantiene en posiciones intermedias también para todas las especies. Los resultados arrojados para $P$. taeda coinciden con los resultados de Hafley y Schreuder (1977) quienes estudiaron el ajuste de diferentes distribuciones en rodales de $P$. taeda, $P$. palustris y P. echinata y concluyeron que $S_{B}$ fue la que mostró mejor ajuste, mientras que Gamma se situó entre las funciones con peores resultados. Por su parte, Gorgoso et al. (2012), trabajando con $P$. pinaster, $P$. radiata y $P$. sylvestris, destacan la superioridad de $S_{\mathrm{B}}$ frente a Weibull. Para todas las especies, el índice de error, coincidió con los resultados del porcentaje de parcelas rechazadas basado en el análisis la prueba de Kolmogorov-Smirnov, para las funciones que mostraron mejor y peor ajuste para cada especie. Sin embargo, para las posiciones intermedias hubo algunos cambios.

\section{CONCLUSIONES}

Los resultados obtenidos en este trabajo verifican la existencia de una fdp para las especies en estudio, la cual ajusta y explica satisfactoriamente la distribución diamétrica de los árboles. Con base en la calidad de ajuste y en el método de estimación de sus parámetros, se propone el uso de la función de distribución de probabilidad de $S_{B}$ de Johnson para modelar la distribución diamétrica de Pinus taeda, mientras que para E. dunnii y E. globulus se recomienda la función Weibull reversa. Las distribuciones seleccionadas proporcionan mayor adherencia a las distribuciones reales. La estimación de los parámetros de estas funciones mediante el método de los momentos brinda resultados satisfactorios en general y puede ser incorporado en sistemas de prognosis.

\section{AGRADECIMENTOS}

A las empresas Global Forest Partners, Cloverly y Bosques del Sur por su valioso apoyo para el desarrollo de este trabajo.

\section{REFERENCIAS}

Abreu E, JR Scolforo, A De Oliveira, JM De Mello, H Kanegae. 2002. Modelagem para prognose precoce do volume por classe diamétrica para Eucalyptus grandis. Scientia Forestalis 61: 86-102.

Al-fawzan MA. 2000. Methods for estimating the parameters of the Weibull distribution. InterStat, statistics on the Internet. Consultado 05 feb. 2014, Disponible en t: URL: http:// www.ip.statjournals.net:2002/InterStat/ARTICLES/2000/ abstracts/O00001.html-ssi

Bailey R, T Dell.1973. Quantifying diameter distributions with the Weibull Function. Forest Science 19(2): 97-104.

Burkhart H, M Tome. 2012. Modelling Forest Trees and Stands. Dordrecht, New York. Springer. 457p.

Eisfeld RL. 2004. Modelagem do crescimento e da produção de Pinus taeda L. por meio de função probabilística e processo de difusão. Dissertação Mestrado em manejo Florestal. Curitiba. Universidade Federal do Paraná. 67 p.

Gadow KV. 1983. Fitting distributions in Pinus patula stands. Pretoria. South African Forestry Journal 126: 20-29.

Garcia O. 1981. Simplified method-of-moments estimation for the Weibull distribution. New Zealand. Journal of Forestry Science 11: 304-306.

Gibbons JG, S Chakraborti. 1992. Nonparametric statistical inference. Third Edition. New York, USA. Dekker. 420 p.

Glade JE. 1986. Prognose de volume por calsse diamétricas para Eucalyptus grandis Hill Ex-Maiden. Tese Maestradno. Curitiba, Brasil. Universidade Federal do Paraná. 94 p.

Gorgoso JJ, A Rojo, A Cámara-Obregón, U Diéguez-Aranda. 2012. A comparison of estimation methods for fitting Weibull, Johnson's SB and beta functions to Pinus pinaster, $\mathrm{Pi}$ nus radiate and Pinus sylvestris stands in northwest Spain. Forest Systems 21(3): 446-459.

Hafley WL, HT Schreuder. 1977. Statistical distributions for fitting diameter and height data in even-aged stands. Canadian Journal of Forest Research 4: 481-487.

Hawkins K, J Hotvedt, Q Cao, B Jackson. 1988. Using the Weibull distribution to model harvesting machine productivity. Forest Products Journal 38(4): 59-65.

Johnson NL, JO Kitchen. 1971.Tables to facilitate fitting SB curves II: both terminals known. Great Britain. Biometrika 
58(3): 657-668.

Kuru GA, AGD Whyte, RC Woollons. 1992. Utility of reverse Weibull and extreme value density functions to refine diameter distribution growth estimates. Forest Ecology and Management 48(1): 165-174.

Liu CH, S Zhang, Y Lei, P Newton, L Zhang. 2004. Evaluation of three methods for predicting diameter distributions of black spruce (Picea mariana) plantations in central.Montreal. Canadian Journal of Forest Research 34: 2424-2432.

Maestri R. 2003. Modelo de crescimento e produção para povoamentos clonais de Eucalyptus grandis considerando variáveis ambientais. Tese Doutorado em Manejo Florestal Pós- graduação em Engenharia Florestal. Curitiba, Brasil. Universidade Federal de Paraná. 143 p.

Methol R. 2001.Comparisons of approaches to modeling tree taper, stand structure and stand dynamics in forest plantation. Ph.D. Thesis. Christchurch, New Zealand. New Zealand School of Forestry, University of Canterbury. 29 p.

Methol R. 2003. SAG grandis Sistema de Apoyo a la Gestión de plantaciones de Eucalyptus grandis. INIA. Serie Técnica 131. p. 4.

Methol R. 2006. SAG globulus Sistema de Apoyo a la Gestión de plantaciones de Eucalyptus globulus. INIA. Serie Técnica 158. p. 34.

Nanang DM. 1998. Suitability of the Normal, Log-normal and Weibull distributions for fitting diameter distributions of neem plantations in Northern Ghana. Forest Ecology and Management 103(1): 1-7.

Nelson T. 1964. Distribution and growth of loblolly pine. Forest Science 10(1): 105-114.

Newnham R. 1992. Variable form taper functions for four Alberta tree species. Canadian Journal of Forest Research 22: 210-223.

Retslaff FAS, A Figueiredo Filho, AN Dias, LG Bernett, MA Figura MA. 2012 Prognose do crescimento eda produção em classes de diâmetro para povoamentos desbastados de Eucalyptus grandis no Sul do Brasil. Revista Árvore 36: 719-732.

Reynolds MJR, T Burk, W Huang. 1988. Goodness-of-fit test and model selection procedures for diameter distribution mo- dels. Bethesda. Forest Science 34(2): 373-399.

Sanquetta CR, A Behling, AP Dalla Corte, S Péllico Netto, AL Rodrigues, AA Simon. 2014. A model based on environmental factors for diameter distribution in black wattle in Brazil. PLoS ONE 9(6): e100093. doi: 10.1371/journal. pone. 0100093

SAS (Statistical Analisys System Institute). 2007. User's guide release 9.2 edition. Cary: SAS Institute Inc.

Scolforo JR. 2002. Biometria Florestal- Manejo Florestal Avançado. Departamento Ciências Florestais, Universidade Federal de Lavras. 311p.

Scolforo JR, A Thierschi. 1998. Estimativas e testes da distribuição de frequência diametrica para Eucalyptus camaldulensis, através da distribuição $\mathrm{Sb}$, por diferentes métodos de ajuste. Scientia Forestalis 54: 93-106.

Shiver BD. 1988. Sample sizes and estimation methods for the Weibull distribution for unthinned Slash pine plantation diameter distributions. Forest Science 34 (3): 809-814.

Stepka T, G Lisboa, S Kurchaidt. 2011. Funções densidade de probabilidade para a estimativa da distribuição diamétrica em povoamento de Eucalyptus sp. na região centro-sul do Paraná. Ambiência Guarapuava 7(3): 429-439.

Vanclay JK. 1994. Modelling forest growth and yield. Applications to mixed tropical forest. Wallingford, UK. CAB International. $312 \mathrm{p}$.

Vallejos OS, CR Sanquetta, JE Arce, S do Amaral, AP Dalla. 2011. Proposta metodológica para o ajuste ótimo da distribuição diamétrica $\mathrm{S}_{\mathrm{B}}$ de Johnson. Revista Árvore 35(1): 151-156.

Vallejos OS, CR Sanquetta, JE Arce, S do Amaral, AP Dalla.2004. Proposta metodológica para o ajuste ótimo da distribuição diamétricaWeibull 3P. Floresta 34(3): 387-393.

Xu L, GR Wood, RC Woollons, AGD Whyte.1992. Stand table prediction with Reverse Weibull and Extreme Value density functions: some theoretical considerations. Forest Ecology and Management 48(1): 175-178.

Zhang L, KC Packard, C Liu. 2003. A comparison of estimation methods for fitting Weibull and Johnson's SB distributions to mixed spruce-fir stands in northeastern North America. Canadian Journal of Forest Research 33: 1340-1347. 